\title{
Synchronous Analysis for Diagnostics of Rolling Bearings in the Turbojet Engine
}

\author{
Zdzisław Gosiewski \\ Department of Mechanical Engineering, Technical University of Bialystok, ul. Wiejska 45C, 15-351 Bialystok, Poland \\ Email: gosiewski@pb.bialystok.pl \\ Andrzej Romaniuk \\ ul. Św. Bonifacego 74 m. 160, 02-936 Warszawa, Poland \\ Email: andrzej.a.romaniuk@wp.pl
}

Received 12 October 2003

\begin{abstract}
Designed in the Aviation Institute, the K-15 turbojet engine has got rolling bearings, which answers with frequency $5.87 \times \omega_{r}$ the unbalance excitation. The signal with such frequency indicates a fault of the outer race of the rolling bearing. A set of the digital synchronous filters was used for the K-15 vibration spectrum analysis. A procedure of filtration was performed by the computer software. The sychronous summation of the measured signals was carried out before the spectrum analysis. Two cases were considered: the engine with a small force due to unbalance (a small angular velocity of the rotor), and the engine with a big unbalance force (high angular velocity). In the first case, the outer race frequency was not observed, despite the existence of the vibration amplitude (caused by unknown disturbances) with such frequency before the synchronous summation. In the second case, the outer race frequency after synchronous summation has enlarged amplitude while other spectrum components in its vicinity have been damped. It underlines the usefulness of the synchronous analysis in the vibration diagnostics of the rotating machinery.
\end{abstract}

Keywords and phrases: diagnostics, nonstationery vibrations, synchronous summation, synchronous filtration, turbojet engine, rolling bearings.

\section{INTRODUCTION}

Proper diagnostics of a rolling bearing condition is of great importance in rotating machinery maintenance. This procedure is based on measurement of vibration level. Generally, the level of vibration generated by the rolling bearing is very low not only in proportion to other sources of vibration but to noise as well. For this reason, the methods of separation of bearing-generated signal must be implemented. The well-known and widely described method is the highfrequency resonance technique which utilitizes a resonance of the bearing-support structure [7]. The support structure plays a role of a mechanical filter. This enables us not only to separate the bearing signal in the frequency domain but to perform amplification as well [9].

The main disadvantage of this method is that in the highfrequency region, the frequency components from various resonances are mixed and as the bandpass frequency of such a mechanical filter is strictly defined, measurement can be

This is an open access article distributed under the Creative Commons Attribution License, which permits unrestricted use, distribution, and reproduction in any medium, provided the original work is properly cited. done for the strictly defined rotating frequency. In the paper, the alternative approach is proposed. We measure the rolling bearing vibration signal directly and simultaneously utilize the information about forces acting in the bearing planes. To improve the signal-to-noise coefficient, the synchronous filtration and the synchronous summation are proposed.

\section{THE CAUSES OF VIBRATION GENERATION}

\subsection{Equations of motion for the rigid shaft with flexible foundation}

In the rotating machinery, most of the external excitations are synchronous to the constant factor with rotor angular velocity $\omega_{r}$. So more, such excitation as the rotor unbalance causes sub- or multisynchronous vibrations of rotating machinery's components, for example, of rolling bearings. So, for proper diagnostics of the bearings, the knowledge of forces acting in the bearing planes is strongly recommended.

The procedure will be explained on the simplified example of a massless shaft with a rigid disk (see Figure 1). The example is rather loosely connected with the K-15 engine but gives a good insight into the proposed procedure. 


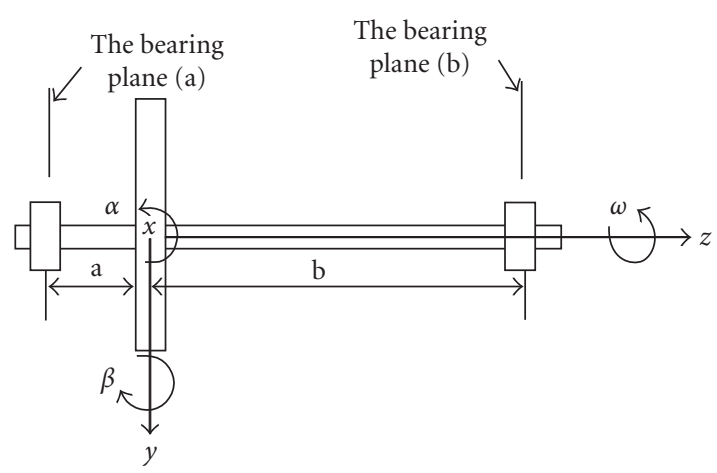

FIgURE 1: Model of the rigid rotor with the flexible foundation.

We can determine the forces by solving differential equations of the rotor motion $[3,5]$ :

$$
\begin{aligned}
m \ddot{x}+ & \left(b_{x a}+b_{x b}\right) \dot{x}+\left(-a b_{x a}+b_{x b}\right) \dot{\beta}+\left(k_{x a}+k_{x b}\right) x \\
& +\left(-a k_{x a}+a k_{x b}\right) \beta=m e \omega_{r}^{2} \cos \omega_{r} t \\
m \ddot{y}+ & \left(b_{y a}+b_{y b}\right) \dot{y}+\left(-a b_{y a}+b b_{y b}\right) \dot{\alpha}+\left(k_{y a}+k_{y b}\right) y \\
& +\left(-a k_{y a}+b k_{y b}\right) \alpha=m e \omega_{r}^{2} \sin \omega_{r} t \\
I \ddot{\beta}+ & I_{p} \omega_{r} \dot{\alpha}+\left(-a b_{x a}+b b_{x b}\right) \dot{x}+\left(a^{2} b_{x a}+b^{2} b_{x b}\right) \dot{\beta} \\
+ & \left(-a k_{x a}+b k_{x b}\right) x+\left(a^{2} k_{x a}+b^{2} k_{x b}\right) \beta \\
& =\left(I_{p}-I\right) \tau \omega_{r}^{2} \cos \left(\omega_{r} t+\beta_{\tau}\right) \\
I \ddot{\alpha}- & I_{p} \omega_{r} \dot{\beta}+\left(-a b_{y a}+b b_{y b}\right) \dot{y}+\left(a^{2} b_{y a}+b^{2} b_{y b}\right) \dot{\alpha} \\
+ & \left(-a k_{y a}+b k_{y b}\right) y+\left(a^{2} k_{y a}+b^{2} k_{y b}\right) \alpha \\
= & \left(I_{p}-I\right) \tau \omega_{r}^{2} \cos \left(\omega_{r} t+\beta_{\tau}\right)
\end{aligned}
$$

where $b_{x a}, b_{x b}, b_{y a}, b_{y b} ; k_{x a}, k_{x b}, k_{y a}, k_{y b}$ are the damping and stiffness coefficients, for the left $a$ and right $b$ support in $x$ and $y$ axes, respectively, $I, I_{p}$ are the axis and the polar moments of inertia, respectively, $m$ is the mass, $e$ is the mass eccentricity, $\tau$ is the the angular misalignment of the principal axis of moment of the rotor with respect to the centerline of the shaft.

Equations (1)-(4) can be expressed in the matrix form

$$
\mathbf{M z}+\mathbf{G} \dot{z}+\mathbf{B z}+\mathbf{K z}=\mathbf{V}
$$

where: $\mathbf{M}$ is the mass matrix, $\mathbf{G}$ is the gyroscopic matrix, $\mathbf{B}$ is the damping matrix, $\mathbf{K}$ is the stiffness matrix, $\mathbf{V}$ is the unbalance vector, $\mathbf{z}$ is the rotor's displacement vector, and $\mathbf{z}=\left[\begin{array}{llll}\mathbf{x} & \beta & \mathbf{y} & -\alpha\end{array}\right]^{T}$.

The rotor motion can also be expressed in terms of the coordinates of the rotor center at the bearing planes, that is, $\mathbf{w}_{b}=\left[\begin{array}{llll}x_{a} & x_{b} & y_{a} & y_{b}\end{array}\right]^{T}$, or by the coordinates of the rotor center at measurement planes, that is, $\mathbf{w}_{m}=\left[\begin{array}{llll}x_{c} & x_{d} & y_{c} & y_{d}\end{array}\right]^{T}$. For algebraic simplicity, it will be assumed that the bearing and measurement planes coincide. The coordinates can be transformed one into another according to the following formula:

$$
\begin{gathered}
\mathbf{w}=\mathbf{T}_{1} \mathbf{z}, \\
\mathbf{T}_{1}=\left[\begin{array}{cc}
\mathbf{T}_{b} & \mathbf{0} \\
\mathbf{0} & \mathbf{T}_{b}
\end{array}\right], \quad \mathbf{T}_{b}=\left[\begin{array}{cc}
1 & -a \\
1 & b
\end{array}\right] .
\end{gathered}
$$

According to virtual work rule, we have $\mathbf{V}^{T} \delta \mathbf{z}=\mathbf{P}^{T} \delta \mathbf{w}$, where $\mathbf{P}$ are unbalance forces in the bearing planes, and $\delta \mathbf{w}=\mathbf{T}_{1} \delta \mathbf{z}$. Finally, we have

$$
\mathbf{V}=\mathbf{T}_{1}^{T} \mathbf{P}
$$

Introducing (6) and (8) into matrix equation (5), we will obtain the motion equations in the coordinates connected with measurement/bearing planes

$$
\mathbf{M T}^{-1} \ddot{\mathbf{W}}+\mathbf{G T}^{-1} \dot{\mathbf{w}}+\mathbf{B T}^{-1} \dot{\mathbf{w}}+\mathbf{K T}^{-1} \mathbf{w}=\mathbf{T}_{1}^{T} \mathbf{P} .
$$

Since the measurements are done in the coordinates $\mathbf{w}$, we can determine the unbalance forces $\mathbf{P}$ acting in the measurement/bearing planes for given angular velocity by solving these differential equations. From the opposite side, when we have unbalance forces, we can calculate (from (9)) displacements for different angular velocities. In the further considerations, we use a signal denoted by $w(t)$ as a contact energy generated by proper component of force $\mathbf{P}$.

\subsection{Model of the bearing vibration signal}

$\mathrm{Su}$ and Lin [9] proposed the following model of the bearing vibration signal:

$$
\begin{gathered}
x_{m}(t)=\int_{-\infty}^{t} d(\tau) w(\tau) a_{m}(\tau) e^{-\alpha_{m}(t-\tau)} \cos \omega_{m}(t-\tau) d \tau \\
X_{m}(\omega)=\left[D(\omega) * W(\omega) * A_{m}(\omega)\right] H_{m}(\omega)
\end{gathered}
$$

where $X_{m}(\omega)$ is a frequency characteristic of the $m$ th (high) mode of vibration, $D(\omega)$ is the Fourier transform of $d(t)$, $d(t)$ is the impulse train (e.g., generated by defected bearing), $W(\omega)$ is the Fourier transform of $w(t), w(t)$ is the weighting function describing the contact energy (e.g., caused by inertia forces generated by rotor unbalance), $A_{m}(\omega)$ is the Fourier transform of $a_{m}(t), a_{m}(t)$ reflects the structural characteristics of the transmission path between the input and output at resonance of $m$ th mode, $H_{m}(\omega)$ is the Fourier transform of $e^{-\alpha_{m} t} \cos \omega_{m} t$, it is the description of bandpass mechanical filter connected with resonance of $m$ th mode, and $\alpha_{m}$ is the damping factor of the bearing-housing ensemble, it can be considered as a quality of mechanical filter.

We analyze the periodic properties of the functions $w(t)$ and $a_{m}(t)$ as proposed by McFadden and Smith [7]. A magnitude of the contact energy $w(t)$ is mainly influenced by the loadings associated with the misalignment or the dynamic unbalance of the shaft, the axial loading, the radial loading, the preload, and the manufacturing imperfections. In Table 1, the periodic properties of different loads $w(t)$ are shown. 
TABLE 1: Periodic characteristics of various loadings and the transmission paths for different defects.

\begin{tabular}{lccc}
\hline Causes of periodicities & \multicolumn{3}{c}{ Periodicities for different defects } \\
\cline { 2 - 4 } Stationary loading & Outer race & Inner race & Roller \\
Loading due to shaft unbalance & $\frac{1}{f_{1}}$ & $\frac{1}{f_{1}}$ & $\frac{1}{\left(f_{1}-f_{\text {cage }}\right)}$ \\
Loadings due to roller errors & $\frac{1}{f_{\text {cage }}}$ & Without periodicity & Without periodicity \\
Transmission path & Without periodicity & $\frac{1}{\left(f_{1}-f_{\text {cage }}\right)}$ & $\frac{1}{f_{1}}$ \\
\hline
\end{tabular}

From (10) and (11), we can deduce that the energy of the signal components with the defect characteristic frequency depends on the weighting function describing the contact energy $w(t)$ and the structural characteristics $a_{m}(t)$ of the transmission path between the input and the output. The energy of the signal components depends on stability of characteristic frequencies of the contact energy and the transmission path between the input and the output. The synchronous filtration, due to changing of the rotating frequency, eliminates nonstability of the characteristic frequencies.

\section{THE ISOLATION OF THE ROTOR AND THE ROLLING BEARINGS SIGNAL COMPONENTS}

\subsection{Synchronous averaging}

The application of the correlation function $R_{x x}(\tau)$ is useful when we do not know the characteristic feature of a signal under investigation. To eliminate or reduce randomness of the digital signal $x(k)$, we can average $x(k)$ by its input autocovariance sequence $R_{x x}(k)$ and its Fourier spectrum $H(\Omega)$-by input autopower spectrum $S_{x x}(\Omega)$, where $\Omega$ is the normalized frequency. In this case, output autocovariance $R_{y y}(k)$ and output autopower spectrum $S_{y y}(\Omega)$ result from estimates $R_{x x}(k)$ and $S_{x x}(\Omega)$ of the input signal, respectively $[1]$

$$
\begin{aligned}
& R_{y y}(k)=h(k) * h(-k) * R_{x x}(k), \\
& S_{y y}(\Omega)=H(\Omega) H^{*}(\Omega) S_{x x}(\Omega),
\end{aligned}
$$

where $h(k)$ is the system impulse response.

The correlation function gives us information about the existence of periodical components, but we are unable to restore these components in a time domain.

By means of synchronous averaging, we can eliminate all nonperiodic signals [2]:

$$
\begin{aligned}
y(t) & =\frac{1}{m_{p}} \sum_{i=0}^{m_{p}}\left[\varphi_{d}(t)+n_{i}(t)\right] \\
& =\varphi_{d}(t)+\frac{1}{m_{p}} \sum_{i=0}^{m_{p}} n_{i}(t) \cong \varphi_{d}(t),
\end{aligned}
$$

where $\varphi_{d}(t)$ is the periodic signal, $n_{i}(t)$ are the nondeterministic components, $m_{p}$ is the number of periods.
This property is of great importance because it allows us to select one-by-one deterministic signals from the measured time sequence.

The synchronous averaging operation is similar to a synchronous filtration. When the number $m$ of periods increases, the averaging signal approaches the periodic signal.

We denote by $T_{s s}$ a total time of average and introduce a frequency of the useful signal

$$
f_{s s}=\frac{1}{T_{s s}}
$$

In some applications, the disadvantage of this procedure is that not only the fundamental component $f_{s s}$ of signal is detected but also the components with frequencies

$$
f_{s s}, 2 f_{s s}, 3 f_{s s}, \ldots \ldots, n f_{s s}, \ldots,
$$

where $n$ is the integral positive number.

For these reasons, synchronous averaging can be applied with full satisfaction only as a preprocessing procedure prior to the synchronous filtration.

The advantage of the synchronous averaging is that a phenomenon of sidebands does not appear. The phenomenon of sidebands is dangerous especially in the case of a signal, which contains components with large levels in proportion to the components of our interest.

\subsection{Synchronous filtration}

A way of vibration signal measurement, which is synchronized with rotor normalized angular velocity $\Omega_{r}$, was presented in [4] for purposes of the automatic balancing system. Nowadays, a development of the computerized calculation methods enables the practical application of the synchronized digital filtration.

A structure scheme of the synchronized vibration measurement path is shown in Figure 2. [8].

In a standard application, the digital filter realizes operations on a data set in which samples are evenly distributed along the time axis, but to obtain phase compatibility, we are forced to scale the localization of samples in time. We can explain this on an example of the impulse response matrix of the bandpass filter [6]:

$$
h(n)=\left(1 \frac{1}{n \pi}\right) \sin \left(n \Omega_{1}\right) \cos \left(n \Omega_{c}\right),
$$




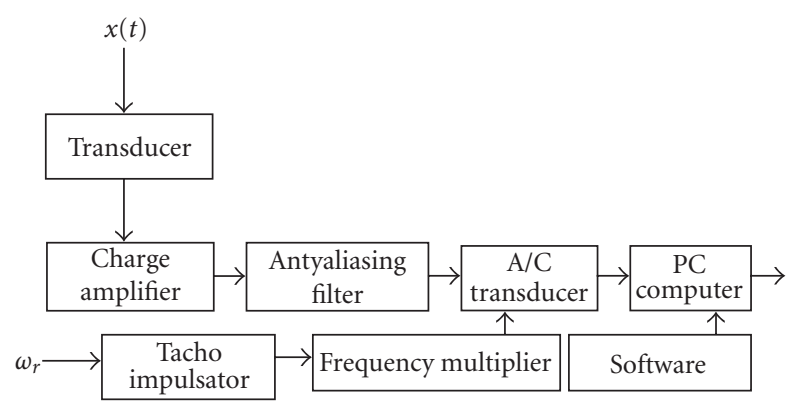

FIGURE 2: Instrumentation setup of the synchronous vibration measurement.

where $\Omega_{c}=f_{c} / 2 \pi f_{d}, f_{c}$ is the center frequency of bandpass filter, $f_{c}=2 \pi \Omega, \Omega$ is a normalized (on the unit circle) frequency, $f_{d}$ is the sampling frequency, $\Omega_{1}$ is the frequency defining the band of the filter. Taking into account frequency correction coefficient $f / f_{c}=k_{f}$, (16) has the form

$$
h(n)=\left(\frac{1}{n \pi}\right) \sin \left(n k_{f} \Omega_{1}\right) \cos \left(n k_{f} \Omega_{c}\right) .
$$

We have to know the coefficient $k_{f}$ to implement the algorithm, which allows us to change frequency of the filter under investigation. This classic realization of the tracking filter is inefficient. We are obliged to continuously measure frequency and after each measurement to change the coefficients of the filter. A delay between frequency measurement and changing the coefficients is the source of error. To eliminate such error, we have introduced the synchronous filtration.

\subsection{Differences between the synchronous filtration and Fourier transform}

We can calculate a frequency spectrum (amplitude and phase) of the signal by using of the synchronous digital filter (SDF). Determination of each frequency line (sample) of the spectrum is realized in the time domain by the single bandpass filter.

The inverse digital Fourier transform (IDFT) algorithm multiplies samples in the frequency domain (which represents frequency spectrum of the input signal we want to restore in the time domain) by IDFT coefficients (which represents frequency spectrum of each filter). Because multiplication in the frequency domain corresponds to convolution in the time domain as an output of the IDTF, we get a signal in the time domain.

We should decrease or increase the number of IDFT coefficients to equal them to the number of input samples. Increasing of the coefficients number allows us to use the greater number of samples in the time domain and leads to the following advantages.

(a) Diminution of a discontinuity impact between the first and the last samples in the time domain. The impulse $\delta$, which represents this discontinuity, is represented in the frequency domain by evenly distributed spectrum (a white noise). The ratio of the white noise to signal diminishes with the increase of the number of the input samples. (b) Detection of low-frequency modulation is possible for the higher resolution in the frequency domain.

The output of the bandpass filter is a sinusoid with changing amplitude. The amplitude change depends on dynamic properties of the input signal and the band of the filter. As far as DFT is concerned, we get the set of sinusoidal components with the constant amplitude and phase shift for every normalized frequency.

DFT analyzes a set of samples taken during the time period given a priori. Shifting the set by one sample in a positive time direction, we can obtain the all-pass filter. We obtain a required transfer characteristic of the desired synchronous filter by the calculation of chosen frequency components. Next, we have the filter output by summation of the components in the time domain, while the characteristic of the digital filter is encoded in its impulse response (filter coefficients).

For a given frequency spectrum, IDFT restores a signal in the time domain, SDF calculates the output signal by a convolution of the input signal with an impulse response of the desired filter. DFT calculates the spectrum components so we can treat it as the set of the bandpass filters. We obtain each frequency component by the multiplication of the transform coefficients by the input signal samples.

\subsection{The comparison of recursive and nonrecursive filters}

\section{Sidebands}

Sidebands are connected only with the nonrecursive filters due to an impulse response truncation. The impulse response truncation is realized by the multiplication of the input signal by a window function. Because the multiplication in the time domain is equivalent to the convolution in the frequency domain, the sidebands occur. Due to the sidebands occurrence, the output signal contains not only the useful components but useless components as well. We can put the useless components into two categories: synchronous and nonsynchronous ones.

The synchronous summation procedure eliminates the nonsynchronous components from the input signal. Elimination of the synchronous components, which frequencies are the multiple of measured signal, can be obtained by designing of special filter algorithm, so these components do not occur in the sidebands. DFT with the inherent rectangular window is an example.

\section{Phase linearity}

The recursive filters introduce the nonlinearity to the phase characteristics.

\section{Memory}

The nonrecursive filters need a finite volume of the processor memory which is a positive feature. They memorize only the number of the impulse samples equal to the number of impulse response coefficients in the filter algorithm. For this reason, a casual distortion signal whips out, after using in the calculation procedure, the number of impulse samples equal 
to the number of impulse response coefficients. The recursive filter due to the recursive character of its algorithm theoretically remembers unlimited number of samples. Using the recursive filter as the synchronous filter, it is not possible to obtain full synchronism, because it is impossible to restrict the number of the input signal samples, which we take to calculate the output sample, to the number equal to impulse response coefficients.

\section{Initial condition}

Using the recursive filter, we always have to remove at the beginning some number of output samples as they are unreliable. The number of removed samples depends on the error we can approve.

\section{The efficiency of the filter algorithm}

For the continuous work with known initial conditions or after transient period, the recursive filters are faster because they realize only a few operations of the multiplication. For interrupted work, their efficiency is similar to the efficiency of nonrecursive filters. For long transient period, their time efficiency may be even worse.

\subsection{Fast algorithms}

In the case of the synchronous filters, it is possible to design a faster algorithm due to the feature that all components are multiple of the first component. Such convolution features like association and commutation allow us to reduce the number of operations. This procedure is possible only for the nonrecursive filters since their principle of operation is based on the convolution.

\section{THE SELECTION METHODS OF THE SIGNAL PROCESSING ALGORITHM}

When frequency of the signal under investigation is known, the synchronous filtration gives much better results than correlation function or the power spectrum density function $S_{x x}$. In this case, our task is to separate deterministic components with known periodicity while all other components should be removed. The determination of the rotor unbalance is a typical practical application.

We can also use the synchronous filtration for rolling bearing diagnostics. In this case, the frequency of the characteristic signal for each part of the bearing is known. Because in this case phase relations are of no importance, we can apply stochastic methods as well. The usage of the nonrecursive filter with a rectangular window combined with the synchronous filtration allows us to remove components whose frequency is the multiple of the band center frequency. This kind of the nonrecursive filter is characterized by the highlevel sideband, so the nonharmonic components are presented in the output signal. We can damp the nonharmonic components in two ways: choosing the window parameters to achieve the low level of the sidebands or applying two filters in a cascade; one with the rectangular window and next with, for example, the Hamming window.

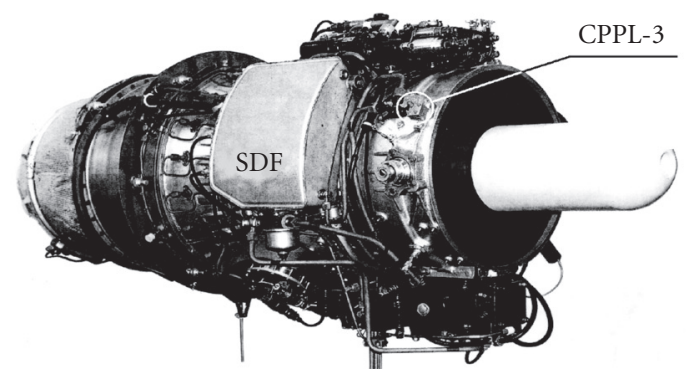

Figure 3: The K-15 turbojet engine with the acceleration transducer CPPL-3.

Application of two connected filters means that we get the impulse response matrix $h(n)$ whose number of coefficients is two times greater than number of coefficients in matrices $h_{1}(n)$ and $h_{2}(n)$. Better solution is to design the filter with the Hamming window, which is able to damp the unuseful harmonics, because in a calculation procedure, it uses two times less coefficients than in the case of two filters in the cascade, so it runs faster, and introduces less phase shift.

A number of impulse response coefficients used as filter coefficients determine the filter frequency resolution. Instead of applying the input signal, the number of samples equal to the filter coefficients number, we can use a reduced number of input samples, by repeating the beginning portion of samples. For example, if the number of samples is reduced four times, we should repeat it four times. This procedure reduces time necessary to measure samples but increases distortion connected with discontinuity of nonsynchronic signals.

Before filtration, we should carry out the synchronous summation on the input signal. The synchronous summation can be executed on the output signal of the tracking filter, in order to enlarge amplitude of synchronous components. The synchronous filter allows us to realize another averaging operation in the time domain. We can calculate the output signal using the number of input samples being the multiple of the filter coefficients.

The other method is the ensemble average of frequency spectrum.

\section{SYNCHRONOUS FILTRATION APPLICATION}

During the test on the engine test stand, a vibration acceleration signal in the radial direction of the K-15 turbojet engine with the single-suction impeller was recorded. The location of the acceleration transducer type CPPL-3 is shown in Figure 3. The aim of the transducer is to measure the overall vibration. The complete procedure with implementation of equations of motion presented in Section 2.1 was not yet performed, the usage of synchronous digital filtration with implementation of four transducers will enable us in the future to calculate the forces acting in the bearings plane.

Figure 4 shows the ensemble average of the frequency spectrum of the vibration acceleration signal. The fundamental synchronous frequency component $f_{1}$ is clearly seen. The multiple of the fundamental frequency and the other 


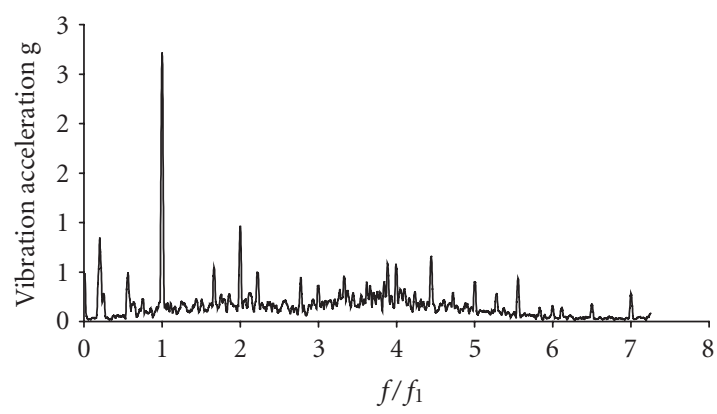

Figure 4: The ensemble average of the frequency spectrum of the vibration acceleration signal radial components, 14.5 samples per revolution.

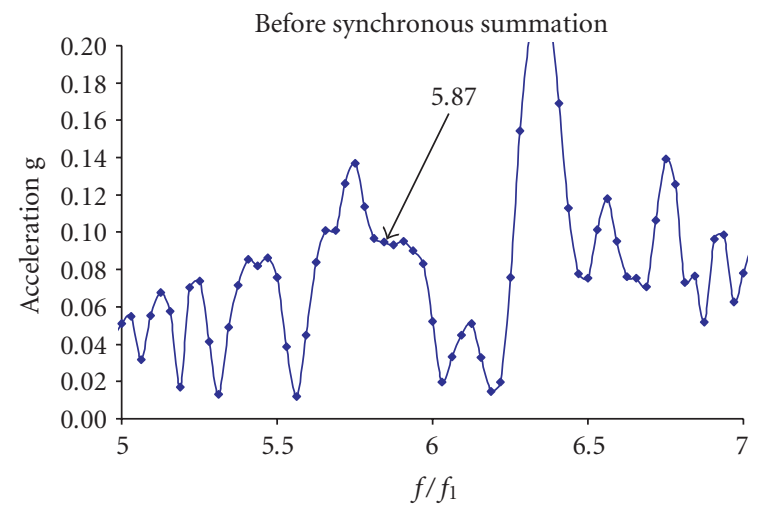

(a)

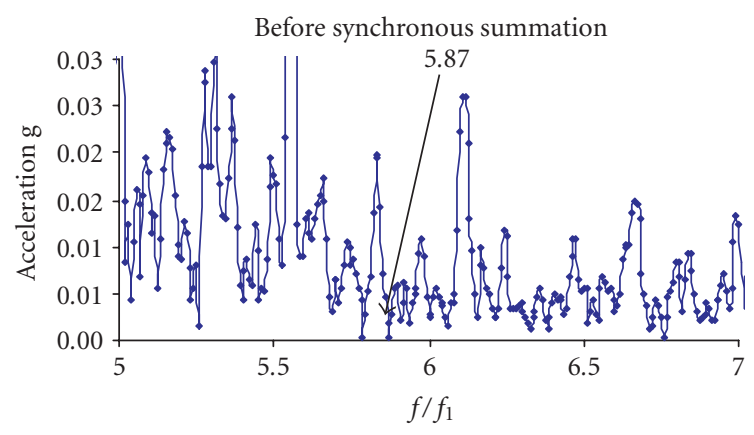

(b)

FIGURE 5: Frequency spectrum. Rotational speed (a) $6857 \mathrm{rev} / \mathrm{m}$, (b) $14500 \mathrm{rev} / \mathrm{m}$. Sampling frequency is 58 samples/rev.

frequencies synchronous with the fundamental component, for example, the $5.87 \times f_{1}$ component generated by the outerrace irregularity of the frontal rolling bearing, are also seen.

The characteristic outer-race frequency of the QBJ55SP5 roller bearing was determined using the manufacturer information.

The frequency analysis by means of the synchronous filter set was carried out. In order to enlarge the amplitude for the characteristic frequency $5.87 \times f_{1}$ of the rolling bearing, the synchronous averaging was performed prior to the fre-

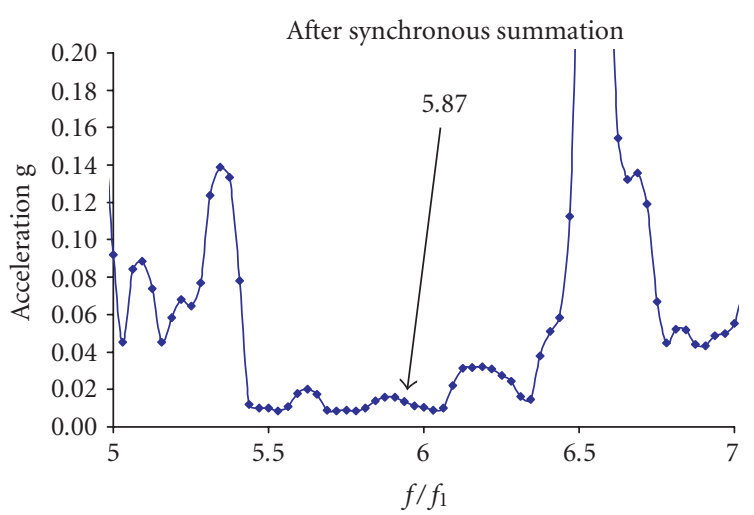

(a)

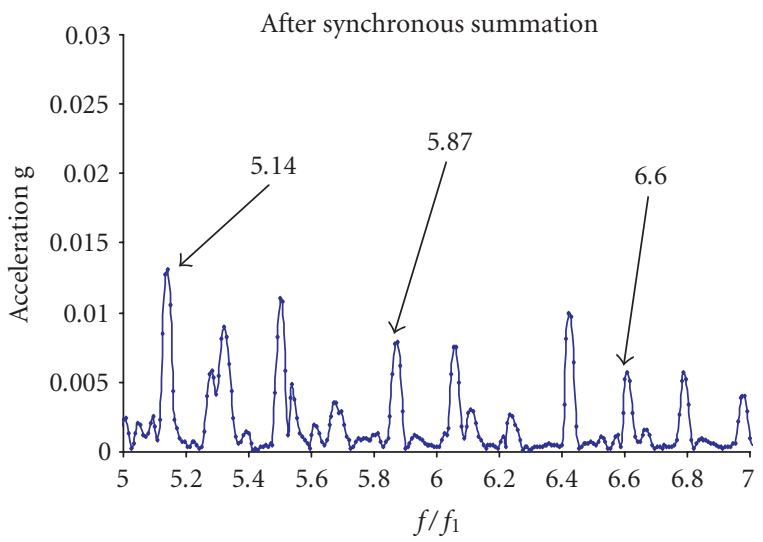

(b)

FIGURE 6: Frequency spectrum after synchronous averaging. Rotational speed (a) $6857 \mathrm{rev} / \mathrm{m}$, (b) $14500 \mathrm{rev} / \mathrm{m}$. Sampling frequency is 58 samples/rev.

quency analysis. The two cases were taken into account: the engine with small unbalance forces caused by relatively small rotor angular velocity, the engine with big unbalance forces caused by high angular velocity. For small unbalance forces (see left part of Figures 5 and 6), the fundamental component $f_{1}$ is hardly seen. In the case of the engine with small unbalance forces, after the synchronous summation, the 5,87 multiple of the rotating frequency was not seen, despite the presence of such a component before the operation of the synchronous summation.

In the case of the engine's vibration signal where the fundamental is dominant (see right part of Figures 5 and 6), after the synchronous summation of the input signal, the 5,87 multiple of rotating frequency has got the enlarged amplitude and the nonsynchronic components in its vicinity are damped.

\section{PRACTICAL IMPORTANCE}

The high-frequency modulation technique is widely considered in rolling bearing diagnostics. The main reason for 
doing this is the necessity of amplification of weak signal generated by bearings. The disadvantage of this technique is that due to modulation property, it is impossible to use this technique when rotating frequency of the measured object is changing because the modulation frequency is determined by the bearing-disk support parameters and we cannot change it. The other disadvantage is that it is difficult to know the amplitude of the measured signal to estimate the dimension of a defect. We cannot estimate the shape of the defect by measuring the vibration signal using the highfrequency resonance technique.

In our approach, the direct low-frequency signal first time proposed by [10] is used. Having both the information of the force which is applied to the bearing and the bearing response, we can estimate the kind and dimension of the defect. The experimental results depend on proper extracting of the signal of interest from distortions so application of synchronous digital filtration improves them. Due to phase linearity of nonrecursive filters, it is possible to realize additionsubtraction operations in the time domain on a measured signal components prior to the digital signal processing.

\section{REFERENCES}

[1] J. V. Candy, Signal Processing: the Modern Approach, McGrawHill, New York, NY, USA, 1988, pp. 83-87.

[2] C. Cempel, Podstawy wibroakustycznej diagnostyki maszyn, Wydawn. Nauk. Techn., Warszawa, Polska, 1982, pp. 74-77.

[3] Z. Gosiewski and A. Muszyńska, Dynamika maszyn wirnikowych, Technical University of Koszalin, Koszalin, Poland, 1992, pp. 38-52.

[4] Z. Gosiewski, Aktywne regulowanie poziomu drgań maszyn wirnikowych ze sztywnym wirnikiem o zmiennym niewyważenie, Ph.D. thesis, IPPT PAN, Warszawa, Poland, 1981, pp. 45-50.

[5] M. Lalanne and G. Ferraris, Rotordynamics Prediction in Engineering, John Wiley \& Sons, New York, NY, USA, 1998, pp. 31-33.

[6] P. A. Lynn and W. Fuerst, Introductory Digital Signal Processing with Computer Applications, John Wiley \& Sons, New York, NY, USA, 1994, pp. 138-144.

[7] P. D. McFadden and J. D. Smith, "Model for the vibration produced by a single point defect in a rolling element bearing," Journal of Sound and Vibration, vol. 96, no. 1, pp. 69-82, 1984.

[8] A. Romaniuk, "Filtr śledzạcy w wibroakustycznej diagnostyce wirnika i łożysk tocznych," Zeszyty Naukowe Politechniki Rzeszowskiej, Nr 186, Mechanika zeszyt 56, Awionika, tom 1, pp. 201-208, 2001.

[9] Y.-T. Su and S.-J. Lin, "On initial fault detection of a tapered roller bearing: frequency domain analysis," Journal of Sound and Vibration, vol. 155, no. 1, pp. 75-84, 1992.

[10] C. S. Sunnersjö, "Rolling bearing vibrations-The effects of geometrical imperfections and wear," Journal of Sound and Vibration, vol. 98, no. 4, pp. 455-474, 1985. 

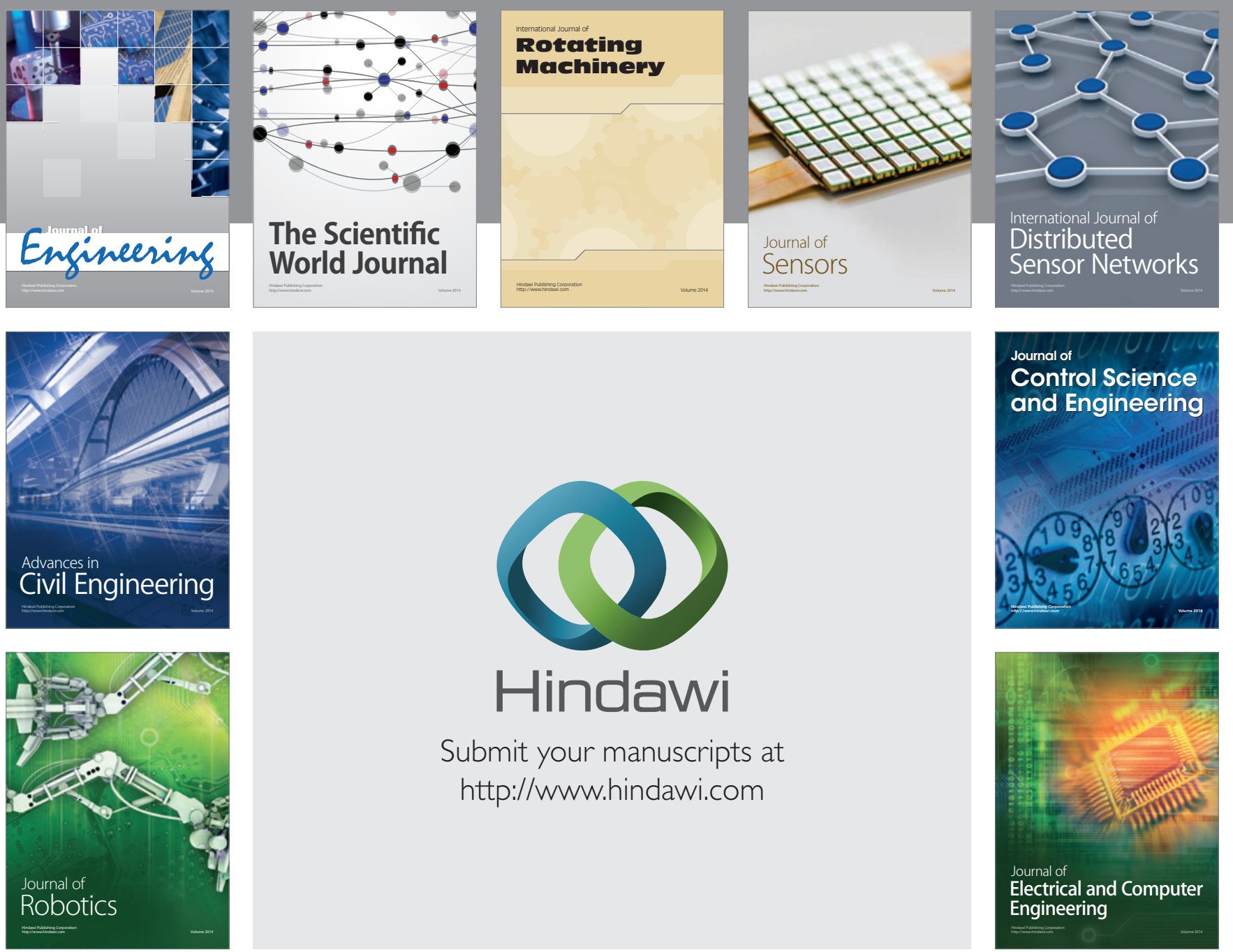

Submit your manuscripts at

http://www.hindawi.com
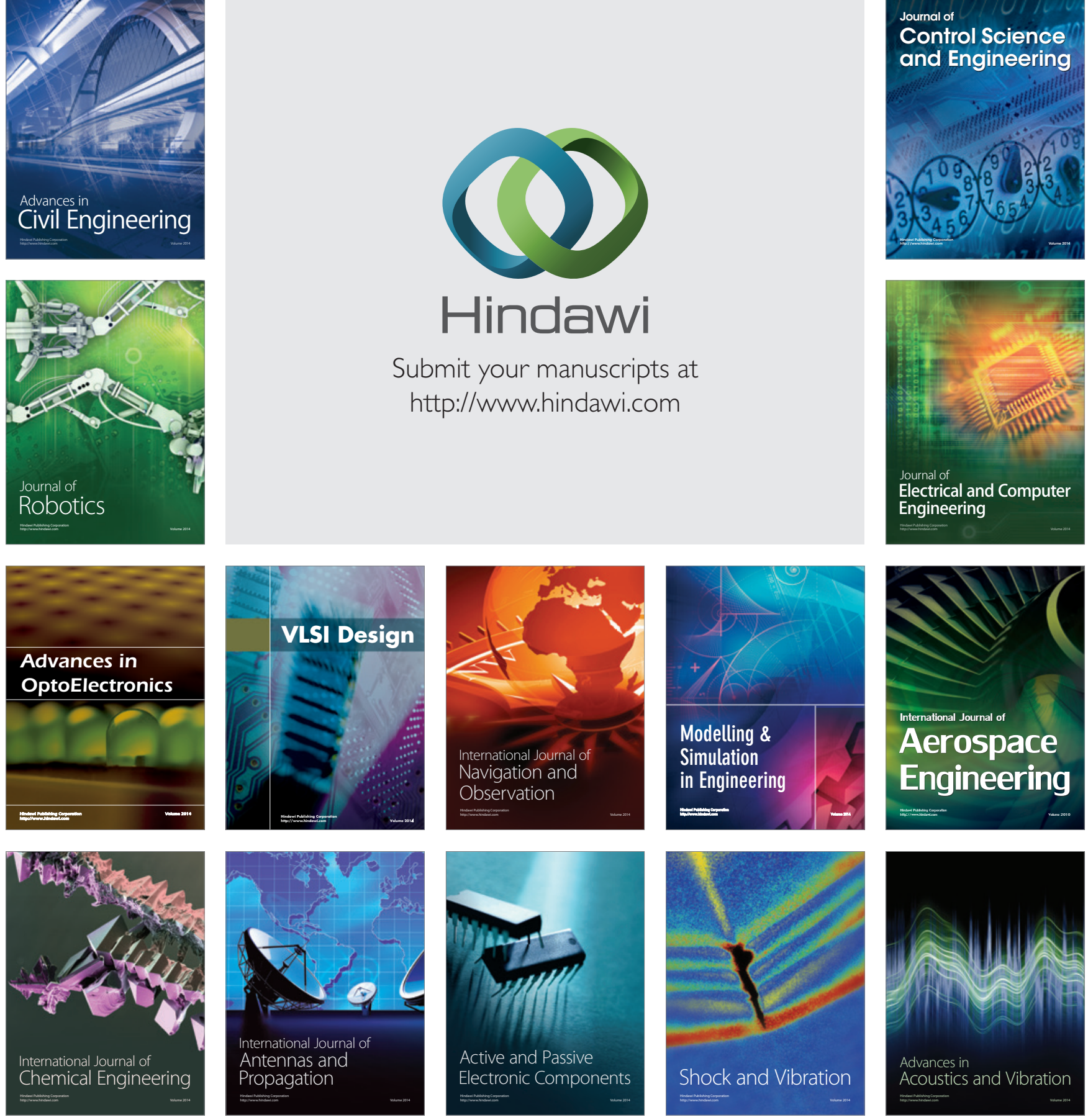